this to mean that star formation took place over an extended region which is nevertheless all associated into what looks like one big loose cluster. This is a much different case than in NGC 330 where star formation took place all within a very small region and therefore probably all at very much the same time.

Westerlund: There are several dense young clusters in the LMC where star formation appears to have continued in the centre after it was over in the outer parts.

\title{
69. THE WING OF THE SMALL MAGELLANIC CLOUD
}

\author{
B. E. Westerlund \\ Mount Stromlo Observatory
}

\section{The Clusters}

It is important to study some of the less bright and less rich clusters in the Magellanic Clouds in order to complete the picture of their populations as derived from studies of their giant clusters and associations. The region around NGC 419

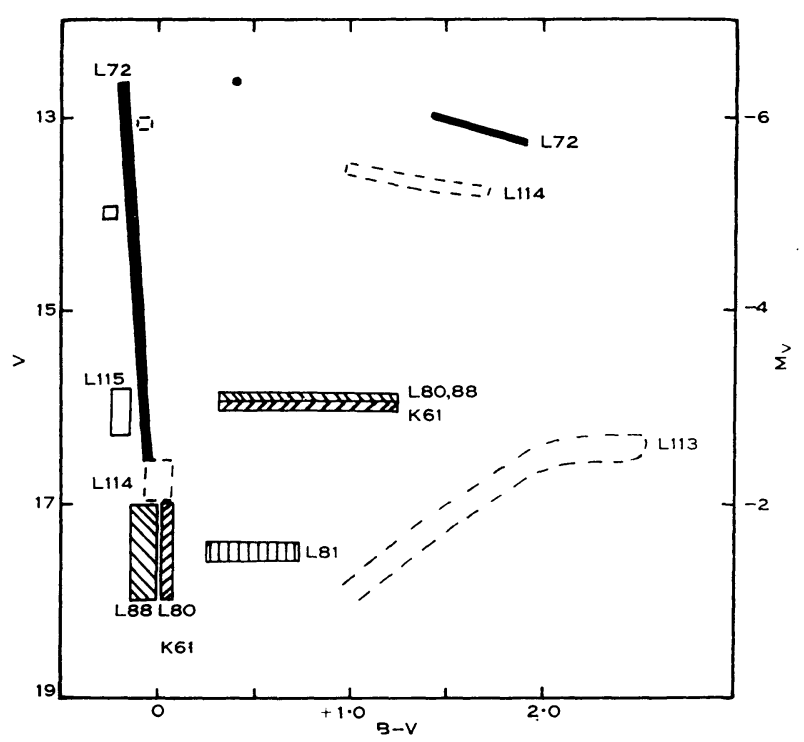

Fig. 1.-Composite colour-magnitude diagram for the blue clusters and for the globular cluster L113.

in the Small Cloud is fairly rich in small- and medium-sized blue clusters and has, in addition, a photoelectric sequence (Arp 1958). Several clusters and groups of stars in this region have now been investigated (Westerlund, unpublished data). The resulting colour-magnitude arrays for four clusters in this region are presented in Figure 1 together with the arrays for three clusters in the wing. The clusters are identified by their numbers in Kron's $(\mathrm{K})$ or Lindsay's (L) catalogues (Kron 1956; Lindsay 1958). 
The arrays for the two clusters L72(=NGC 376) and L114 (in the wing) agree in all main features with the arrays for NGC 330 (Arp 1959b). A similar agreement is found for the clusters L80, L88, and K61 when compared with NGC 458 (Arp 1959a). The composite diagram shows how the Hertzsprung gap shrinks with decreasing luminosity of the red and yellow giant stars of the clusters. Finally, it practically disappears as indicated here by the preliminary data for the cluster L81.

Clusters of the type represented by NGC 458 may thus be fairly common in the Small Cloud. We may then expect to find the corresponding kind of stars among the field stars in the main body of the Cloud. Thus, stars of intermediate colours and fainter than $V=\mathbf{1 6}$ mag cannot be disregarded immediately as galactic foreground stars in studies of the population of the Clouds. It appears extremely important to establish accurately the frequency of clusters of this age group, $3 \times 10^{7}$ to $10^{8}$ years, in various parts of the Clouds. Similarly, a determination of the proportion of field stars of intermediate colours would be extremely valuable.

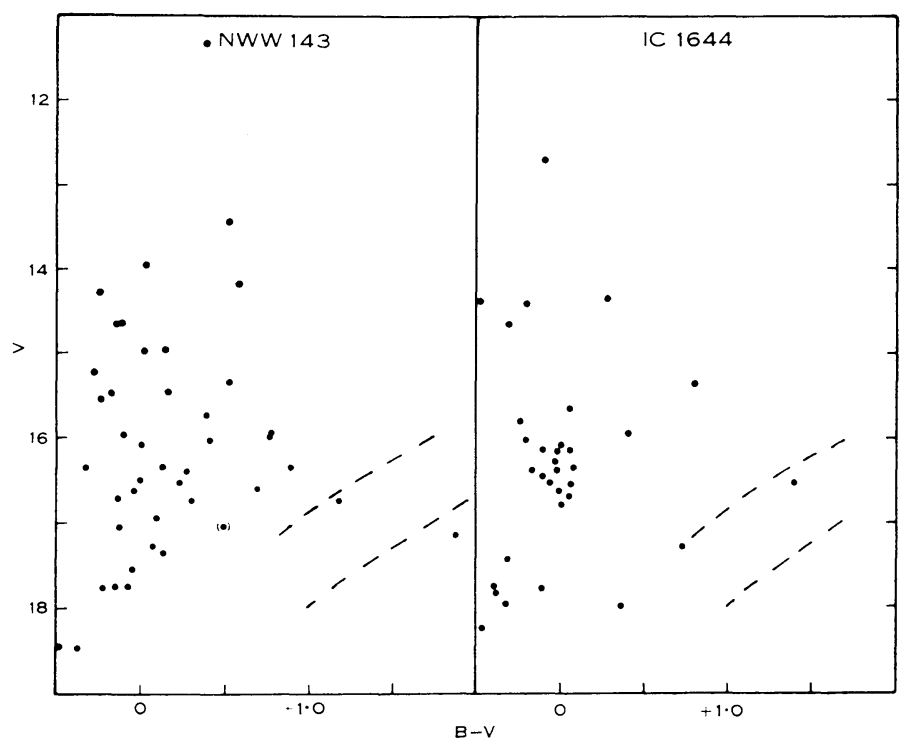

Fig. 2.-Colour-magnitude diagrams for NWW 143 and IC 1644.

On our survey plates the two emission nebulae, NWW 143 (Nail et al. 1953) and IC 1644, appear as nuclei in more extensive and fairly symmetrical weak nebulae centred on what appear to be blue clusters. The colour-magnitude diagrams for these groups of stars are shown in Figure 2. It is difficult to draw any conclusions about the group of stars in NWW 143. There is no doubt in the case of IC 1644 that the stars form a young cluster. The brightest object, at $V=12 \cdot 7$, is the nucleus of IC 1644 itself. It shows similarities to the central star in 30 Doradus and the whole system may perhaps be looked upon as a miniature of the 30 Doradus complex.

The globular cluster L113 is superposed on the wing of the Small Cloud. It is situated about $4^{\circ}$ from the centre of the Small Cloud and appears to belong to its 
halo population. Its giant branch (Fig. 1) is similar to the one in NGC 121 (Tifft 1963) with regard to the apparent magnitudes of the brightest giants as well as to their colours. Of the three stars with $B-V>+2 \cdot 0$, at least one is variable.

\section{The Field Stars in the Wing}

In a recent study of the region of NGC 602 (Westerlund, unpublished data) slightly over 900 stars have been measured to a limiting magnitude of $V=20$ mag. The colour-magnitude diagram for the field stars in the region is reproduced in Figure 3. We note a well-defined main sequence clearly separated from the yellowred stars above $V=19$ mag. The luminosity function of the blue stars agrees well with the Salpeter-Sandage zero-age luminosity function.

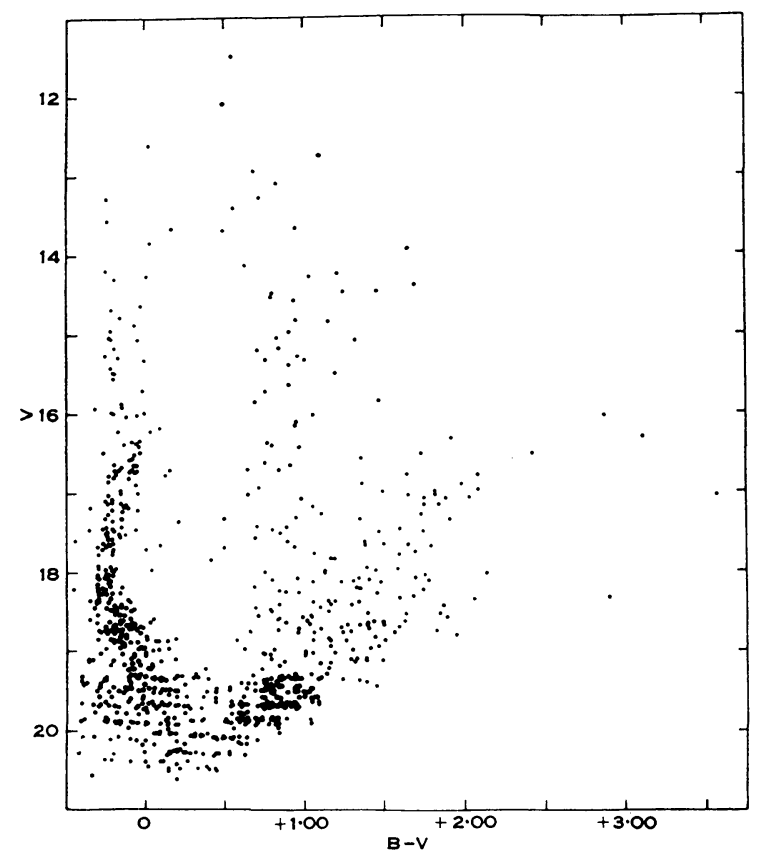

Fig. 3.-Colour-magnitude diagram for the field stars in the region of NGC 602 .

Most of the yellow and red stars are probably galactic foreground stars. The total number observed to $V=20$ mag is 425 whereas we would expect about 400 (area of 0.061 square degrees) according to van Rhijn's tables (Allen 1955). A few of the faint yellow and red stars may be members of the halo of the Small Cloud. This is to be expected from the appearance of globular clusters at still greater distances from the centre of the Cloud, but hardly any of these stars are members of the wing. It may be significant that no "connecting bridges" of stars of intermediate colours appear for $V<19$ mag. Similar results have been obtained previously for the region of NGC 456-465 (Westerlund 1961). 
At still greater distances from the centre of the Small Cloud, in the region of the clusters L113, L114, and L115 we have found several blue field stars with magnitudes between $V=14$ and 16. In this region eight bright supergiant stars have also been observed and their membership in the Cloud established from spectra as well as from their $\mathrm{H} \beta$ absorption (Westerlund, Danziger, and Graham 1963).

\section{The Emission Nebulae}

On long-exposure plates taken with the Uppsala Schmidt telescope in $\mathrm{H} \alpha$ light of the Small Cloud we have identified several very extensive weak emission nebulae which have not been listed previously. Their exciting groups of blue stars have been investigated; samples have been given above. We have continued the investigation by studying the dimensions of these nebulae. Two examples will be given here:

(a) Very Weak Large Strömgren Sphere Centred at $01^{h} 10^{m},-72^{\circ} 50^{\prime}$ (1975).There are several blue stars inside this nebula which has a radius of about $80 \mathrm{pc}$. Most of the excitation can, however, be ascribed to one star in its centre with the magnitude $V=13 \cdot 7$. The estimated emission measure of 2000 gives a density of $N_{\mathrm{H}}=3 \cdot 5$ atoms per $\mathrm{cm}^{3}$. The total mass of ionized hydrogen is then about $2 \times 10^{5}$ solar masses.

(b) The Nebulosity Surrounding NGC 602.-We will divide this nebulosity into (i) the previously known nebula, NGC 602 itself, and (ii) a faint arc possibly emerging from NGC 602 but apparently centred on another association of blue stars, L107.

(i) The excitation of the nebula is mainly due to the three brightest stars in the association in NGC 602 with $M_{V} \sim-5 \mathrm{mag}$. The radius of the nebula is $25 \mathrm{pc}$ and its mean density is $N_{\mathrm{H}}=13$ (Aller, unpublished data). The mass of the nebula is then about $2 \times 10^{4}$ solar masses.

(ii) The arc centred on L107 has a radius of about $150 \mathrm{pc}$, a thickness of $40 \mathrm{pc}$, and an observed length of about $150 \mathrm{pc}$. Most of the excitation is due to one star in Ll07 with $M_{V}=-6$ mag. The comparison of the brightness of the arc with that of NGC 602 indicates a density of about $N_{H}=3$. The mass of the arc is then about $3 \times 10^{4}$ solar masses.

It is somewhat surprising that no emission is observed between this arc and the association L107. Obviously, the density of hydrogen is too low there for detection as nebulosity on our plates. If we assume a density of 0.6 atoms per $\mathrm{cm}^{3}$ (emission measure about 100) inside a sphere with the radius $r=150 \mathrm{pc}$ we derive a total mass of hydrogen of about $2 \times 10^{5}$ solar masses. This possible but unobserved mass may be compared with the total mass of stars in the region of about $10^{4}$ solar masses.

The values derived here for the masses of the weak emission nebulae in the Small Cloud are comparable with values for the bright small nebulae in the Large Cloud. This is in agreement with Henize's (1956) conclusions that nebulosity is as abundant in the Small Cloud as in the Large, but the interstellar medium of the Small Cloud does not attain such dense concentrations as does the interstellar medium of the Large Cloud. However, as the shape of several of the weak emission nebulae in the Small Cloud indicate that they are expanding, we may possibly add that the 
difference between the nebulae in the two Clouds is an age effect: the nebulae in the Small Cloud are generally older and have had time to expand more so that their densities are approaching the density of the interstellar medium.

Finally, attention is called to a small emission nebula in the wing of the Small Cloud, about $6^{\circ}$ from the centre of the Cloud. It has been independently identified by Lindsay (1961) and Westerlund (Westerlund and Henize 1963). It is centred on a 15th magnitude star and has a radius of about $1.2 \mathrm{pc}$. The density of the nebula is $N_{\mathrm{H}}=70$, giving it a total mass of about 12 solar masses. The mass of the star is about 24 solar masses. No nebulosity has been observed anywhere in the neighbourhood of this object and it appears difficult to understand its existence if it has not been ejected from the star. Therefore, we suggest that this star is evolving through gas ejection. The age of the star is about $10^{7}$ years. If we assume a rate of mass ejection of $10^{21} \mathrm{~g} / \mathrm{sec}$, equal to that estimated by Pagel (1958) for $\mathrm{P}$ Cygni, we find that ejection must have taken place at this rate for $8 \times 10^{5}$ years and that the average expansion rate must be $1.5 \mathrm{~km} / \mathrm{sec}$.

\section{References}

Allen, C. W. (1955)._-“Astrophysical Quantities." 1st Ed. p. 213 (Univ. London, Athlone Press: London.)

ArP, H. C. (1958).-A.J. $63: 273-82$.

ARP, H. C. $(1959 a)$. - A.J. 64: 175-82.

ARP, H. C. $(1959 b)$. -A.J. $64: 254-8$.

HeNIze, K. G. (1956).-Ap. J. Suppl. 2: 315-44.

KroN, G. E. (1956).-P.A.S.P. 68: 125-30.

LINDSAY, E. M. (1958).-M.N. 118: 172-82.

LiNDSAY, E. M. (1961).—A.J. 66: 169-85.

Nail, Virginia McKibben, Whitney, Ch. A., and Wade, C. M. (1953).-Proc. Nat. Acad. Sci. (Washington) 39: 1168-76= Harvard Reprint No. 377.

PAgel, B. E. (1958)._-“Etoiles à Raies d'Emission." [Liège.] Mém.Soc. Roy.Sci. Liège (4) 20: $177-92$. TiFfT, W. G. (1963).-M.N. (in press).

Westerlund, B. E. (1961).-Uppsala Obs. Ann. 5 : No. 2.

Westerlund, B. E., Danziger, I. J., and Graham, J. (1963).-Observatory 83: 74-9.

Westerlund, B. E., and Henize, K. G. (1963).-P.A.S.P. (in press). Also Abstract in A.J. 68: 299 .

\section{Discussion}

de Vaucouleurs: I would like to comment on the wing region especially on the unusual nature of the more distant star cloud (Shapley's wing).

Thackeray: Are your supergiants without visible surrounding gas classified by Graham as earlier or later than B2?

Westerlund: The earliest is B0 Ia and the latest is A2 Ia. They agree exactly with those in the Galaxy in the $(B-V)$ and $(U-B)$ diagram.

Gascoigne: Does that mean they are unreddened?

Westerlund: I assume so.

Pagel: How was the mass of the gaseous shell estimated?

Westerlund: We applied two methods; one used the ratio of intensities in the $3727 \AA$ doublet and the other $\mathrm{H} \alpha$ measurements; both methods were used to derive the final result. 